\title{
¿Cómo enseñamos a los futuros docentes? análisis documental y contraste de percepciones entre alumnos y profesores
}

\author{
How do we teach future teachers? Documentary analysis and contrast \\ between students and teachers perceptions \\ David Hortigüela Alcalá, ${ }^{a}$ Ángel Pérez Pueyo, ${ }^{b}$ Alberto Moreno Doña

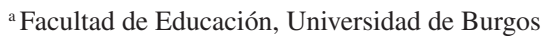 \\ Telf.: 947259517. Correo electrónico: dhortiguela@ubu.es \\ ${ }^{\mathrm{b}}$ Facultad de Ciencias de la Actividad Física y el Deporte, Universidad de León. \\ Telf.: 987293001. Correo electrónico: angel.perez.pueyo@unileon.es \\ ${ }^{\mathrm{c}}$ Universidad de Valparaíso \\ Telf.: (56) 32 2603832. Correo electrónico: alberto.moreno@uv.cl
}

\begin{abstract}
En el presente artículo se analizan las categorías de implicación en la evaluación, la autonomía del alumno hacia el trabajo y la implantación de estrategias didácticas que derivan de la titulación del Grado de Educación Primaria de la Facultad de Educación de la Universidad de Burgos, España. Participaron 122 alumnos y 15 docentes de la titulación. Se ha empleado una metodología cualitativa en la que se utilizan tres instrumentos de recogida de datos: a) análisis de 20 guías docentes de asignaturas, b) técnica grupal para animar la conversación con los alumnos y c) grupo de discusión con los docentes. Los alumnos manifiestan que el aprendizaje es más elevado y útil cuando se articulan procedimientos evaluativos que les permitan implicarse en el proceso. Los profesores presentan desacuerdos sobre el nivel formativo y evaluativo que ha de tener el enfoque de enseñanza que ha de utilizarse.

Palabras clave: implicación en evaluación, autonomía, estrategias didácticas, grupo de discusión, análisis documental.
\end{abstract}

\section{ABSTRACT}

In this article the categories of involvement in the evaluation, learner autonomy to work and the implementation of teaching strategies are analyzed. The study was conducted in the Primary Education Programme at the Faculty of Education in the University of Burgos, Spain. 122 students and 15 teachers of the programme participated. We used a qualitative methodology in which three data collection instruments were used: a) analysis of 20 subjects teaching guides, b) group technique to encourage conversation with students c) group discussion with teachers. Students demonstrate that learning is higher and more useful when evaluative procedures, that enable them involved in the process, are articulated. Teachers have disagreements about the training and evaluative level that the teaching approach to be used must have.

Key words: involvement in evaluation, autonomy, teaching strategies, group discussions, document analysis. 


\section{INTRODUCCIÓN}

En una sociedad tan cambiante como la actual e inmersa en una variedad de perspectivas éticas, sociales y de valores, la educación se convierte, más que nunca, en la herramienta más potente para generar cambios positivos e influyentes a medio plazo (Frederiksen y Beck, 2013). Es por ello que el rol del maestro es clave para orientar, guiar y reconducir los intereses y capacidades de los alumnos que deriven en la adquisición de aprendizajes sólidos asociados al empleo de herramientas aplicables en su día a día. Como indican Powers y otros (2012), los alumnos se encuentran expuestos a una gran cantidad de estímulos como las nuevas tecnologías, redes sociales, etc., que unidos a una situación socioeconómica diversa, desequilibrio en el entorno familiar, inestabilidad y movilidad laboral, etc., provocan que sea necesario utilizar nuevos enfoques de enseñanza que favorezcan la responsabilidad y toma de decisiones desde el principio del proceso formativo (Pang, 2010).

Esto se vincula directamente con el rol que ha de desempeñar la formación inicial del profesorado, ya que en función de cómo los docentes universitarios enseñen a los futuros maestros, estos pueden reproducir, en mayor o menor medida, dichas estrategias en el aula (Ní Chróinín y O’Sullivan, 2014). Pero incluso si se acepta que una parte de los docentes universitarios realiza enormes esfuerzos por llevar a cabo estrategias de enseñanza adecuadas, en muchas ocasiones es habitual que tras su formación inicial enseñen como ellos aprendieron en su etapa de alumnos o imiten a compañeros con más experiencia (Marcelo y Vaillant, 2009). Estos aspectos, que ya comentaban hace varias décadas Fernández-Pérez (1989) o Tejedor (1998), con la implantación completa del Espacio Europeo de Educación Superior, no parecen haber cambiado demasiado.

Por lo tanto, más que la mera impartición de contenidos, la enseñanza ha de estar orientada a la adquisición de recursos de enseñanza, la construcción de la identidad profesional y, sobre todo, al resurgimiento de la labor vocacional del maestro, tan degradada actualmente (Köpsén, 2014). Para ello, es fundamental que desde el ámbito universitario se reflexione acerca de los fines que han de regir las titulaciones de educación, proponiendo procesos de enseñanza activos y adaptados a las demandas sociolaborales e intereses de los alumnos (Lindell, 2006). Esto permitiría huir del "inmovilismo didáctico y pedagógico que predomina en las facultades y centros de formación inicial del profesorado (FIP) y del nefasto efecto que esto genera en los niveles no universitarios del sistema educativo" (Palacios y López-Pastor, 2013, p. 284). No se trata de cambiar totalmente los enfoques, sino de renovar pautas metodológicas y evaluativas que rompan el hermetismo e inconcreción que en algunos casos caracterizan a las guías docentes de las materias. ¿Es posible y coherente que en una asignatura de tres meses y medio lectivos se establezca que el alumno vaya a contribuir a 14 competencias? ¿Por qué no existe una linealidad, concordancia y progresión pedagógica en los tipos de herramientas de aprendizaje e instrumentos de evaluación que se demandan a los alumnos en cada uno de los cursos? Como indican Van der Klink, Boon y Schlusmans (2007), parece más razonable abarcar menos campo de actuación para ser más concreto en los fines que se persiguen, sobre todo, en aras de buscar una secuencia lógica en los cursos y asignaturas de cara a la adquisición de competencias docentes.

Otra de las principales problemáticas inherentes a los procesos formativos iniciales del profesorado radica en la desconexión que en muchos casos existe entre lo que se enseña y 
lo verdaderamente aplicable al aula de infantil y de primaria (Downing y Dyment, 2013). No parece ser demasiado funcional trabajar sobre teorías, principios filosóficos de la educación o leyes educativas si luego eso no se manifiesta en la aplicación de recursos de enseñanza, trabajo con materiales diversos y tratamiento de estrategias que favorezcan el desarrollo cognitivo, social y emocional del niño (Diamond, 2010). Es por ello que el tipo de metodología empleada y el enfoque evaluativo se relacionan directamente con la capacidad del alumno para regular su trabajo, y como consecuencia deriva en la adquisición de una mayor autonomía a la hora de solventar las tareas solicitadas (Hortigüela, PérezPueyo y López-Pastor, 2015).

Por ello, si aceptamos que la implicación del docente no siempre es eficaz en el proceso de enseñanza y aprendizaje de la FIP, y que uno de los problemas es la desconexión que percibe el alumnado entre lo que le han enseñado y la utilidad de esto, parece fundamental atender a las percepciones y valoraciones de los agentes educativos implicados en él (Jang, 2011). El estudiante ha de tener voz y poder participar activamente en aquello que se le enseñe. Es preciso que exista un equilibrio, en la medida de lo posible, entre aquello que pretende enseñar el profesor y las inquietudes e intereses del alumno, buscando puntos de acuerdo que favorezcan su motivación e implicación. En este sentido, algunos estudios como el de Waterman et al. (2010) demuestran que cuando se aplican procesos dialógicos y compartidos en el ámbito universitario los resultados académicos son más elevados y las valoraciones del alumno más satisfactorias. Hendry y Tomitsch (2014) indican que una de las claves se encuentra en acordar, desde el comienzo, unos criterios de evaluación claros, coherentes, que sean comprendidos por el alumno y sobre todo que se asocien a metas factibles a mediano plazo. Esto permite que el alumno tenga claros sus objetivos, el modo de proceder y cuáles son las consecuencias del nivel de implicación y de trabajo realizado (Beck, Skinner y Schwabrow, 2013).

En esta misma línea, y más en la formación inicial del profesorado, la docencia ha de alejarse de la generación de conocimiento a partir de la impartición de contenidos específicos y descontextualizados de la materia, ya que el trabajo emocional y de valores posee una relevancia sustancial en la definición de la futura identidad profesional (Hagenauer y Volet, 2014). Algunas experiencias de trabajo en grupo como la de Ennen, Stark y Lassiter (2015) demuestran cómo los alumnos universitarios que recibieron una formación emocional integrada en las materias mostraron una mayor satisfacción hacia el aprendizaje recibido y una transferencia más directa de esa formación a sus procesos docentes. Además, estudios recientes (Castro et al., 2015) muestran la importancia que tiene el desarrollo de las emociones en el niño como medio para extraer el potencial de sus capacidades de aprendizaje, influyendo favorablemente en su desarrollo psicoevolutivo.

La principal contribución del estudio es el análisis exhaustivo de las estrategias metodológicas y evaluativas que utilizan los docentes que imparten docencia en la formación inicial del profesorado. Su enfoque cualitativo, a través de análisis de guías docentes, técnicas grupales para animar la conversación con alumnos y grupos de discusión con profesores, arroja datos que permiten comprender y reflexionar sobre la transcendencia de estos procesos formativos y cuál es su situación actual. La investigación tiene dos objetivos principales. El primero, analizar los apartados de metodología y evaluación de veinte guías docentes de las titulaciones de Grado en Maestro de Primaria. El segundo, contrastar las percepciones de profesores y alumnos a través de las técnicas grupales para animar la conversación y un grupo de discusión estructurado. Para ello se utilizan las categorías de 
la implicación en la evaluación, la autonomía del alumno hacia el trabajo y la implantación de las estrategias didácticas utilizadas.

\section{MATERIAL Y MÉTODOS}

\subsection{PARTICIPANTES}

Este apartado se estructura a partir de los tres ejes principales del análisis del estudio: guías docentes, percepción del alumnado y percepción del profesorado.

Se han analizado veinte guías docentes de la titulación de Grado de Educación Primaria en la Facultad de Educación de la Universidad de Burgos, España. Se han distribuido por cursos de manera proporcional, eligiéndose cinco de cada uno de los cuatro que constituye el grado. Las asignaturas estudiadas (15 obligatorias y 5 troncales) forman parte de los Departamentos de Didácticas Específicas y de Ciencias de la Educación. Estas guías han sido seleccionadas atendiendo al criterio de muestreo aleatorio por conglomerados, partiendo de los criterios iniciales de la proporcionalidad por cursos y de la preponderancia de las asignaturas obligatorias.

En la técnica grupal para animar la conversación participaron 122 estudiantes, divididos en subgrupos, que se encontraban finalizando cuarto curso del Grado de Educación Primaria. Los alumnos matriculados en esa promoción eran 143, de los que se contó con un $85,3 \%$ de la población total. De ellos, 73 eran chicas y 49 chicos. La media de edad era de $24.32 \pm 2.38$ años. A un $84,3 \%$ de los alumnos únicamente les restaban entre dos y tres asignaturas para finalizar la titulación, entre ellas el trabajo fin de grado, perteneciente al octavo semestre.

En el grupo de discusión de profesores participaron 15 docentes de la titulación, 8 mujeres y 7 hombres. Su media de edad es de $46.13 \pm 7.31$ años y tienen una media de 14.34 años de experiencia. De ellos, 11 son doctores y los otros cuatro Licenciados. El muestreo ha sido intencionado, buscando la representatividad de los Departamentos, la variedad en las áreas a las que pertenecen y su vinculación con las guías docentes que han sido analizadas.

\subsection{INSTRUMENTO}

Para el análisis de las guías docentes se ha empleado una plantilla con una serie de indicadores que valoran los apartados de metodología de enseñanza y aprendizaje y el sistema de evaluación. Estos criterios (Cuadro 1) se estructuran de forma abierta, con el fin de que los investigadores incorporen la mayoría de información relevante en cada campo. Los tres primeros pertenecen al apartado de metodología y los tres segundos al sistema de evaluación empleado. 
Cuadro 1. Indicadores utilizados para la valoración de las guías docentes analizadas

1. Total de horas presenciales y de trabajo que se dedican a las clases teóricas y prácticas.

2. Competencias que se relacionan con los trabajos, informes y pruebas de evaluación realizadas por el alumno.

3. Importancia que se le otorga a las exposiciones orales y al trabajo en grupo.

4. Peso en la calificación que se le otorga al examen.

5. Criterios mínimos que se establecen para garantizar la evaluación continua.

6. Condiciones establecidas para la evaluación excepcional.

En relación a los alumnos, se empleó una técnica grupal para animar la conversación como instrumento de recogida de la información. Se plantearon cuestiones vinculadas con las tres categorías del estudio: implicación en la evaluación, autonomía desarrollada y aplicación de estrategias didácticas. Al ser un grupo muy numeroso se empleó la técnica de generación de subgrupos previos para la organización secuencial de las preguntas y respuestas (Tortorella, Viana y Fettermann, 2015). Se realizaron seis subgrupos de unos veinte estudiantes, de tal modo que tras plantear una pregunta al gran grupo, cada uno, con un portavoz elegido, anotaba las respuestas que posteriormente eran comentadas en voz alta. Eso permitió tanto mantener el orden en la dinámica como la garantía de una reflexión conjunta por todos los participantes (Robinson y Mendelson, 2012). Las preguntas que se realizaron se pueden observar en Cuadro 2.

Cuadro 2. Guion básico del grupo de discusión realizado a los alumnos

1. ¿Estáis satisfechos con los sistemas de evaluación que habéis recibido en la asignatura? ¿Por qué?

2. En general, ¿Se os ha dejado formar parte en la toma de decisiones de los procesos evaluativos de los que habéis formado parte?

3. ¿Se ha fomentado en las asignaturas vuestra autonomía hacia el aprendizaje? ¿Cómo?

4. ¿Os consideráis ahora más capaces, con más recursos y más independientes a la hora de desarrollar una clase con garantías en el aula? ¿Por qué?

5. ¿Os han motivado las estrategias didácticas que habéis recibido en las materias? ¿Cuáles son las ideales para vosotros?

6. ¿Existe transferencia a vuestras clases como futuros docentes de la metodología y contenidos que os han enseñado?

En el grupo de discusión que se realizó con los docentes se utilizaron los procedimientos más comunes de las entrevistas grupales abiertas (Calhoun y Taub, 2014), ya que los docentes tienen una amplia experiencia en la temática del estudio. Además, aunque todos 
los profesores imparten docencia en la titulación del Grado en Primaria, pertenecen a diferentes áreas, algo muy positivo por el carácter multidisciplinar inherente (Calderón, 2011). Las preguntas que se realizaron atienden a las tres categorías del estudio (Cuadro 3).

Cuadro 3. Guion básico del grupo de discusión realizado a los docentes

1. ¿Qué grado de importancia le otorgáis al sistema de evaluación empleado para el aprendizaje del alumno?

2. ¿Procuráis que el alumno tome decisiones a lo largo del proceso evaluador de la asignatura? ¿Cómo?

3. ¿Qué métodos empleáis para generar en el alumno autonomía y responsabilidad en cada una de las tareas demandadas?

4. ¿Qué tipo de retroalimentación utilizáis con los alumnos a lo largo del proceso? ¿La consideráis importante?

5. ¿Qué elementos fundamentales han de tener las estrategias didácticas que utilizáis con los futuros maestros?

6. ¿Tenéis en cuenta la aplicabilidad de las técnicas de enseñanza que utilizáis con los futuros maestros para su labor docente a corto plazo?

Se observa cómo existe una linealidad y relación directa entre los tres instrumentos de recogida de datos empleados. Todos ellos derivan de los dos objetivos del estudio y de las categorías de análisis de la investigación.

\subsection{DISEÑO Y PROCEDIMIENTO}

La investigación constó de cuatro fases bien diferenciadas:

- $\quad$ Fase 1. Estructura de la investigación y contacto con participantes: una vez que se decidió el enfoque cualitativo que se iba a utilizar en la investigación, se delimitaron los objetivos del estudio y se articuló tanto la técnica para animar la conversación de los estudiantes como el grupo de discusión a llevar a cabo, se contactó a los participantes. En relación a los estudiantes, y debido a que uno de los investigadores impartía docencia en cuarto curso, se les convocó en dos sesiones: una primera para explicarles su importancia y el rol a desempeñar en el estudio y otra para llevarse a cabo la conversación. Respecto a los docentes, y al ser compañeros de trabajo en la Facultad, se les informó por email de las pretensiones de la investigación y de su disponibilidad para realizar el grupo de discusión.

- Fase 2. Selección y análisis de las guías docentes: tras haber contactado a los participantes, se seleccionaron las 15 guías docentes objeto de estudio. Se estableció la plantilla con los indicadores a valorar (Tabla 1) y se procedió a su análisis. Se utilizó una plantilla para cada una de las guías y en ella se registró la mayor cantidad de información posible en los apartados de metodología y evaluación, que posteriormente fue comparada y saturada.

- Fase 3. Puesta en práctica de la técnica de conversación con los estudiantes y los grupos de discusión y triangulación de la información: en primer lugar se realizó la conversación con los alumnos, que tuvo una duración aproximada de dos horas. Este, debido a la gran cantidad de participantes, se llevó a cabo en el salón de grados. Al ser alumnos de tres grupos: A, B y C, muchos de ellos no se conocían, por lo que se procedió a realizar una 
presentación previa. Esto no influía en el estudio, ya que la totalidad había cursado las mismas asignaturas de la titulación. Posterior a la conversación con los estudiantes se llevó a cabo el grupo de discusión con profesores. En este caso todos se conocían entre ellos. Se utilizó un aula normal para su desarrollo, teniendo el proceso una duración de 90 minutos. Todos los docentes participaron varias veces, facilitando el intercambio de ideas y la profundización en la información.

Previo consentimiento de los participantes, las conversaciones fueron grabadas en video. Esto facilitó el volcado de información y la realización de categorías comunes para el análisis.

- $\quad$ Fase 4. Categorización de la información y análisis en profundidad de los resultados: toda la información recabada de las guías docentes, las conversaciones con estudiantes y el grupo de discusión con profesores, fue analizada exhaustivamente bajo criterios de saturación de la información y triangulación entre los instrumentos. Tras ello se generaron las tres categorías comunes para el análisis de resultados, estableciendo codificaciones para identificar la proveniencia de los datos. Por lo tanto, se plantea inicialmente un modelo de lógica deductiva que posteriormente repercute en un planteamiento inductivo de generación de categorías que agrupan los resultados obtenidos.

A todos los participantes se les garantizó el anonimato y la confidencialidad de los datos, asegurándoles que sus opiniones únicamente estarían sujetas a los fines de la presente investigación.

\subsection{ANÁLISIS EMPLEADO}

El enfoque del estudio es cualitativo, ya que su principal fin es comprender los fenómenos inherentes a los procesos que constituyen los fenómenos educativos (Svensson y Doumas, 2013), en este caso la formación recibida por los futuros maestros. No se busca la generalización de los datos, sino dar respuesta a interrogantes que emanan de contextos específicos (Ells, 2011), buscando el porqué de las situaciones acontecidas y estableciendo procedimientos de mejora.

Los datos se obtienen a partir de las experiencias vivenciadas por los agentes implicados en el proceso (alumnos y profesores), así como de los documentos (guías docentes) que estructuran y dan identidad al ámbito de trabajo. Se entiende que la riqueza de la información proviene del análisis de multitud de percepciones, que es lo que permite otorgar multidisciplinariedad y profundizar en el estudio (Michie, 2013).

Para garantizar la credibilidad, transferibilidad y confiabilidad se utilizaron pautas de análisis comunes para los tres instrumentos de recogida de datos fundamentadas en las categorías del estudio. Los investigadores adoptan un rol interno e integrado en el estudio, ejerciendo de moderadores en cada una de las conversaciones y en el grupo de discusión. En el análisis del texto se utilizan patrones cruzados coincidentes, lo que favorece la codificación y contabilización de los extractos de texto generados (Saldaña, 2009). Además, tal y como indican Strauss y Corbin (2002), se utilizan los procedimientos de la "Grounded Theory" basados articulación y fragmentación de toda la información recabada en cada una de las categorías. Los resultados se estructuran en tres categorías, atendiendo a un proceso de codificación abierto, axial y selectivo. Se utiliza la saturación como premisa previa para cribar la información, lo que favorece la contabilización de los extractos de texto y la presentación de aquellos más significativos. 


\subsubsection{Generación de categorías y su categorización}

Se ha empleado el software de análisis de texto WEFT QDA, lo que ha permitido, una vez volcados los datos, la generación de tres categorías para estructurar los resultados. Estas categorías subyacen de la temática del estudio, de los dos objetivos de la investigación y de cada uno de los instrumentos de recogida de datos.

Estas categorías son

- Sistemas de evaluación e implicación del alumnado: se analiza todo lo referente a los métodos evaluativos utilizados, sobre todo incidiendo en la forma en la que se favorece que el alumno participe en su toma de decisiones. Se da cabida a los tipos de procedimientos e instrumentos de evaluación de las asignaturas, el feedback existente entre el profesor y el alumno a lo largo del proceso y la claridad de los criterios de calificación establecidos para cada una de las tareas.

- Generación de autonomía en el alumno durante el proceso de enseñanza: se analizan aspectos vinculados con la responsabilidad que el alumno tiene en las asignaturas y las mejoras experimentadas en cuanto a la obtención de recursos, aplicación de los mismos y búsqueda de pautas aplicables a la gestión de tareas de forma independiente.

- $\quad$ Relación de estrategias didácticas utilizadas y su aplicación en el aula: se incide en el tipo de metodologías empleadas por los docentes a lo largo de la carrera y la aplicabilidad y utilidad que pueden tener estas en el aula cuando ejerzan su rol de maestros.

La vinculación de la información a cada categoría favorece una mayor especificación de la misma, y como consecuencia un análisis más exhaustivo, ajustado y comprensivo de los datos (Talburt, 2004).

\subsubsection{Codificación de instrumentos de recogida de datos}

Con el fin de identificar cada extracto de texto con los participantes y el instrumento de recogida de los datos se utilizan acrónimos. Para la información derivada de las guías docentes se utiliza "análisis de guías docentes" (AGD). Para la relativa a las "conversaciones con alumnos" se utiliza (GDA) y para la referente al "grupo de discusión de profesores" se emplea (GDP).

\section{RESULTADOS}

Los resultados se presentan a partir de las tres categorías generadas. Mediante el análisis de patrones cruzados se presenta el número de extractos literales de texto resultantes en cada categoría, mostrándose aquellos más significativos y coincidentes.

\subsection{SISTEMAS DE EVALUACIÓN E IMPLICACIÓN DEL ALUMNADO (273 EXTRACTOS DE TEXTO)}

Respecto a las guías docentes se observa cómo solamente en cinco de las veinte queda explícita la realización de procesos de evaluación formativa. Además, en la gran parte de ellas únicamente se presta atención a la porcentualización de los procedimientos de evaluación, no dejando claro ni los criterios de calificación ni los instrumentos a utilizar. 
En nueve de las guías se indica que es necesario superar cada uno de los procedimientos de evaluación establecidos para superar la convocatoria ordinaria, sin embargo, solamente en tres se asocia la convocatoria extraordinaria con procesos de carácter formativo:

"Los procesos de evaluación formativa no aparecen explícitos en la mayoría de las guías docentes [...]. Solamente en cinco se relacionan estos sistemas con las pruebas evaluativas que se van a desarrollar". "Los criterios de calificación son escasos y apenas aportan información [...]. Hay que intuir qué instrumentos se utilizarán". "En la mayoría de las guías, el apartado de evaluación excepcional se reduce a explicar las pruebas que tendrán que superarse”. (AGD).

Los alumnos manifiestan que todavía en muchas asignaturas la calificación del examen sigue teniendo el mayor peso de la nota, lo que provoca la memorización una semana antes de los contenidos delimitados por el profesor y su olvido poco tiempo después. Valoran más positivamente cuando se les deja tomar parte en el proceso evaluador a través de coevaluaciones o autoevaluaciones, siempre que el profesor lo incentive y también se implique. Agradecen el trabajo de rúbricas y escalas de valoración a lo largo del proceso, ya que les permite conocer de manera real cómo van realizando el trabajo y cómo pueden mejorarlo, alejándose su calificación final de criterios más subjetivos y menos comprensivos:

"Es cierto que cada vez hay más asignaturas en las que si vas a clase no tienes que hacer examen, algo que se agradece [...] Sin embargo en la mayoría el examen es casi lo que decide la calificación". "Con otros profesores hemos coevaluado el trabajo de los compañeros antes de su entrega final, lo que permite mejorarlo". "Al principio no lo entendíamos muy bien, pero finalmente es bueno el uso de rúbricas para saber cómo vas”. (GDA).

Los profesores muestran disparidad de opiniones respecto al rol que ha de desempeñar la evaluación a lo largo del proceso, y sobre todo en la manera en la que se puede incluir al alumnado en la misma. Tres profesores conciben el rol de la evaluación formativa como prioritario en la enseñanza universitaria, ya que es más importante el proceso de guiado y motivación que la mera impartición de contenidos. Sin embargo, cinco de los docentes parecen confundir la implicación del alumno en la evaluación con facilitar demasiado las cosas para que consigan un aprobado:

"Los contenidos están a disposición del alumno a través de un click, [...] Lo verdaderamente interesante es que seamos capaces de generar procesos motivadores que impliquen al alumno sobre aquello que le queremos enseñar". "A estas alturas el alumno ya debería de saber si se quiere implicar o no, lo que no podemos es dárselo todo masticado con la evaluación [...]. Luego nos quejamos". (GDP).

\subsection{GENERACIÓN DE AUTONOMÍA EN EL ALUMNO DURANTE EL PROCESO DE ENSEÑANZA (231} EXTRACTOS DE TEXTO)

Doce de las veinte guías docentes incluyen el trabajo en grupo y la responsabilidad hacia las tareas como competencias directamente asociadas a la metodología. En cuatro guías se delimitan los criterios mínimos que el alumno tendrá que superar al acabar la asignatura, incluyendo los tipos de recursos que se van a llevar a cabo para conseguirse. Sin embargo, el resto solamente describe las competencias sin asociarlas a recursos de trabajo tangibles: 
"Es destacable que la mayoría de las competencias docentes delimitadas en las guías se relacionan con las responsabilidad del alumnado en la superación de los trabajos propuesto". "Se destaca como una carencia la indefinición de criterios de cada uno de los procedimientos de evaluación delimitados". (AGD).

La mayoría de los alumnos manifestaron que en muchos casos no se fomenta la autonomía en las asignaturas, ya que las tareas que se piden casi siempre se ciñen a un trabajo en grupo, exposiciones orales y realización de un examen. Los estudiantes reconocen que en muchas asignaturas solamente hacen lo que se les pide para aprobar, ya que no encuentran la motivación necesaria para aprender cosas relacionadas. Valoran positivamente las dinámicas de grupos y las reflexiones en conjunto que vayan más allá de la impartición de clases magistrales:

"Al final la autonomía depende de uno mismo y de lo que se quiera aprender [...]. En muchas de las asignaturas siempre te suelen pedir el mismo tipo de tareas, solo que en algunas son mucho más exigentes que otras". "Verdaderamente como más se aprende es profundizando en temáticas que verdaderamente interesan, lo mismo que si te piden hacer un buen trabajo, pero no el típico que haces rápido para salir del paso". (GDA).

Por su parte, seis de los docentes manifiestan en el grupo de discusión que su rol no se ha de ceñir a la generación de motivación y autonomía en el alumno, ya que en estudios superiores estos aspectos deberían estar implícitos en la actitud del estudiante, y más en profesiones tan vocacionales como la de maestros. Por el contrario, el resto de los profesores consideran que su rol en esta titulación siempre ha de dirigirse a despertar motivación en el alumno y explorar nuevos campos que incentiven al alumno a aprender en el día a día:

"Suficientes maestros hay ya sin motivación en las aulas como para que nosotros tengamos que invertir tiempo de la asignatura en esto [...]. Son muchos los contenidos los que tenemos que impartir cada vez en menos tiempo". "Personalmente pienso lo contrario, si ya existen maestros poco vocacionales y solamente impartimos contenidos vamos a seguir contribuyendo a ello". (GDP).

\subsection{RELACIÓN DE ESTRATEGIAS DIDÁCTICAS UTILIZADAS Y SU APLICACIÓN EN EL AULA (241 EXTRACTOS DE TEXTO)}

Solamente en tres guías docentes se hace explícito el tipo de estrategias concretas que se va a utilizar, ya que en el resto únicamente se hacen descripciones globales y genéricas como "participación en clase" o "aportaciones y actitud en el aula". En cinco de las guías se comenta la relación que existe entre el tipo de actividades a trabajar y su vinculación directa con el aula de primaria:

"Se echa en falta que se especifiquen técnicas concretas de enseñanza asociadas a los contenidos y la metodología desarrollada". "Se abusa de frases demasiado genéricas que no aclaran ni aportan datos concretos sobre el tipo de metodología empleada". "En pocas guías se vinculan estrategias con su aplicabilidad como docentes". (AGD). 
Existe pleno consenso en el alumnado a la hora de reconocer que las asignaturas más interesantes son aquellas que se relacionan con recursos aplicables que puedan implantarse en el aula, tanto en su fase de prácticas como en su futuro desarrollo profesional. Muestran un mayor desinterés por las asignaturas que se estructuran únicamente a partir de la memorización de contenidos y el estudio de teorías y filosofías. Agradecen especialmente la formación relativa a la innovación en el aula, las técnicas de recolección de información para mejorar los procesos de investigación-acción, y la generación de recursos de enseñanza vinculados al empleo de las nuevas tecnologías:

“A mí lo que más me interesa es saber cómo puedo desarrollar tareas en clase cuando sea maestra [...]. "Poder tener recursos de tareas, actividades, cómo mantener el control o la atención de los niños". "Investigar en el aula para mejorar en el día a día es algo muy útil, lo mismo que el uso de las tecnologías de la información y de la comunicación”. (GDA).

La mayoría de los profesores considera relevante vincular las estrategias que se enseñen en las asignaturas con lo que los futuros maestros van a poder aplicar. No obstante, tres docentes afirman que no hay que convertir las asignaturas en medios con fines utilitaristas, ya que se trata de dar recursos al alumnado para construir su propia manera de dar clase. En esta línea, dos docentes mantienen la postura de que no es necesario innovar en las estrategias didácticas, ya que lo importante es enseñar como siempre se ha hecho:

"Lo que tenemos que tener claro es que si formamos a futuros docentes hay que pensar lo que aporta eso a la creación de la futura identidad profesional". "Nuestro rol no ha de cambiar tanto, que parece que la cosa va por modas, una cosa es que lo que nos toca enseñar y otro lo que les puede interesar a cada uno". (GDP).

\section{DISCUSIÓN}

Se han obtenido datos de verdadera relevancia en relación a los procedimientos de enseñanza articulados, instaurados y asumidos tanto en las guías docentes como en la percepción de estudiantes y profesores. La mayoría de las guías son ambiguas en el tipo de evaluación que se va a llevar a cabo, únicamente se especifica con claridad los porcentajes de calificación que se asignan a cada tarea. Por su parte, los alumnos de último curso del Grado de Educación Primaria destacan la importancia de que les enseñen recursos innovadores y motivantes de transferibilidad al aula. Los docentes presentan desacuerdos sobre cuál debe de ser su rol en las asignaturas, discrepando entre guiar y orientar al alumno e impartir contenidos específicos de la materia.

Respecto a la primera categoría relativa a la implicación del alumno en los procesos de evaluación, se demuestra cómo en las guías docentes apenas se detalla el tipo de evaluación que se llevará a cabo, otorgándose el mayor peso de este apartado a la descripción de tareas asociadas a un porcentaje. Este hecho se vincula directamente con la estructura tradicional de la enseñanza universitaria, donde prima más el qué tengo que hacer para aprobar que los procesos formativos de verdadero aprendizaje (Duncan y Arthurs, 2012). Parece lógico, por tanto, que en la presente investigación los alumnos vean como algo común y poco motivante que el examen siga siendo el principal instrumento de calificación de las materias. Lebec 
y Kesteloot (2015) indican que esta tendencia no es tan predominante como hace diez años, pero que todavía impera la memorización de contenidos como fuente más utilizada para la "acreditación" del aprendizaje. Cherepinsky (2011) refleja que el hecho de utilizar exámenes como instrumento de evaluación no es algo negativo, ya que la realización de esta prueba puede asociarse a procesos formativos previos muy interesantes que hacen más consciente al alumno de sus propios errores. En consecuencia, los estudiantes valoran como recurso de interés por su aplicabilidad la utilización de rúbricas de autoevaluación y coevaluación para registrar el trabajo, algo que les hace implicarse y ser más conscientes de lo realizado. Experiencias en el uso de estos procesos (Ferrandiz-Vindel y Jimenez, 2011) muestran que los alumnos son muy partidarios de coevaluar al compañero, siempre y cuando eso no se traduzca en una calificación.

En la segunda categoría del estudio, relacionada con el fomento de la autonomía del alumno en el proceso de enseñanza, se han obtenido datos relevantes. Si bien es cierto que las competencias delimitadas en las guías docentes se asocian a la responsabilidad del alumno y al desarrollo de motivación hacia la enseñanza, la mayoría de ellas carece de la delimitación de criterios básicos que contemplen la manera de adquirir estas competencias. Esto suele traducirse en una falta de claridad de criterios abiertos y conocidos por el alumno, lo que suele repercutir en una opacidad de la información, en inseguridad del estudiante en la realización de tareas y en mayores reclamaciones del alumno ante la falta de juicios objetivos (Poole, 2013). Los estudiantes han mostrado cierta desmotivación hacia el tipo de tareas que se les solicita en las asignaturas, ya que en muchos casos se repiten y no se aprende nada nuevo. Parece imprescindible que exista una coordinación entre los docentes de una misma titulación, por lo menos por cursos, a la hora de especificar los procedimientos de evaluación, ya que de lo contrario pueden cometerse incoherencias en los procesos de enseñanza que hagan que se debilite en gran medida la formación (Jensen, 2011). En este sentido, los alumnos del estudio valoran como más interesantes las tareas vinculadas a la reflexión sobre temáticas educativas actuales. Por otro lado, los docentes difieren acerca de la manera en la que hay que generar motivación en el aula, ya que unos consideran que ello no es parte del rol del profesor, mientras otros consideran lo contrario. Experiencias de éxito en otros países (Harrinson, 2014) demuestran cómo en la formación inicial del profesorado, al no tratarse de una titulación técnica, lo verdaderamente importante es abordar tareas vinculadas a la generación de actitudes positivas hacia la profesión.

La tercera categoría de análisis referente al empleo de estrategias didácticas en el aula corrobora algunos de los datos obtenidos con anterioridad. Solamente en cinco de las guías se hace referencia a la relación existente entre los contenidos a abordar y su aplicabilidad en el aula. Esto parece algo escaso, ya que ha quedado patente cómo los futuros maestros valoran en mayor medida las asignaturas que, o bien se desarrollan en centros educativos reales o en contextos directamente relacionados (Callejo, 2008). En la presente investigación se asienta esta idea, ya que los estudiantes reconocen que el exceso de clases magistrales de determinados profesores hace que se convierta en prescindible acudir al aula. En este sentido, la mayoría de los docentes entiende que aquello que se enseña tiene que tener una vinculación con lo que el futuro docente aplicará en su aula. Sin embargo, dos de ellos asocian esto con fines utilitaristas de la educación. Este planteamiento parece un error, ya que el papel actual de la docencia universitaria ha de tener una estrecha relación con el trasfondo sociocultural presente en la sociedad (Välimaa y Nokkala, 2014). 


\section{CONCLUSIONES}

En relación al primer objetivo del estudio se concluye que los apartados de metodología y evaluación de las guías docentes analizadas no reflejan de manera detallada los aspectos relativos a las estrategias metodológicas y evaluativas a emplear, únicamente dan peso a los porcentajes de los procedimientos de evaluación que son calificados.

Respecto al segundo objetivo, queda de manifiesto que los alumnos valoran positivamente que se les deje ser partícipes de su evaluación a través de coevaluaciones y el uso de rúbricas a lo largo del proceso. Además, consideran de mayor interés el empleo de estrategias didácticas estrechamente relacionadas con contextos educativos y la realidad docente. Por otro lado, los docentes muestran disparidad de criterios a la hora de entender la evaluación en el aula y la autonomía que conlleva para el alumno, confundiendo en algunos casos la aplicación de estrategias didácticas activas con la facilitación a la hora de obtener una calificación positiva.

El principal aporte de esta investigación ha sido contrastar, mediante un enfoque cualitativo, las valoraciones que tienen los alumnos del último curso de Grado en Primaria con la de los docentes de la titulación respecto a los factores que influyen directamente en la formación inicial del profesorado. Esto se ha relacionado con la información que se atribuye en las guías docentes a la metodología y los sistemas de evaluación, algo novedoso en la literatura actual sobre la temática. El artículo presenta algunas limitaciones. En primer lugar, únicamente se atiende a una titulación, por lo que en futuras investigaciones se podría contrastar con otras como la de infantil. Además, el trabajo se enfoca en una universidad en concreto, por lo que sería de gran interés que se contrastaran estos datos con otros obtenidos en diferentes instituciones. En segundo lugar, los resultados se enmarcan en la docencia de carácter presencial, por lo que parece oportuno realizar investigaciones que demuestren en qué medida influye la asistencia a clase en la formación inicial del profesorado en comparación con estudios universitarios on-line.

Consideramos el artículo de especial interés para todos aquellos docentes universitarios que impartan docencia en titulaciones vinculadas al ámbito educativo, ya que les permitirá profundizar y reflexionar sobre los modelos pedagógicos utilizados actualmente en la formación de los futuros docentes. También puede ser de utilidad para todos aquellos responsables de Vicerrectorado Académico o de Profesorado, ya que a partir de esta temática surge una gran cantidad de posibilidades a la hora de impartir cursos de formación que deriven en la articulación de procesos que puedan garantizar una conexión directa entre la estructura de las guías docentes, las competencias a desarrollar y los intereses del alumnado.

Es evidente que existe una gran indeterminación sobre cuáles han de ser los fines fundamentales que articulan la formación inicial del profesorado, algo que es percibido por los agentes implicados en el proceso. Es por ello que la coordinación docente a la hora de delimitar procedimientos comunes que garanticen la adquisición de competencias docentes se convierte en uno de los retos actuales del ámbito universitario.

\section{REFERENCIAS BIBLIOGRÁFICAS}

Beck, R.J., Skinner, W., \& Schwabrow, L.A. (2013). A Study of Sustainable Assessment Theory in Higher Education Tutorials. Assessment \& Evaluation in Higher Education, 38(3), 326-348. 
Estudios Pedagógicos XLII, N 3: 207-221, 2016

¿CÓMO ENSEÑAMOS A LOS FUTUROS DOCENTES? ANÁLISIS DOCUMENTAL Y CONTRASTE DE PERCEPCIONES ENTRE ALUMNOS Y PROFESORES

Calderón, J.L. (2011). How-To Data Collection Series: The Evolution of the Focused Discussion Group-From Non-Participant to One of the Crew. Qualitative Report, 16(1), 308-311.

Calhoun, D.W., \& Taub, D.J. (2014). Mentor Relationships for Entry-Level Men in Student Affairs. Journal of Student Affairs Research and Practice, 51(2), 183-194.

Callejo, D. (2008). Teacher Education and Research: Imagining Teacher Education between Past and Future. American Educational History Journal, 35(1), 19-40.

Castro, B., Halberstadt, A.G., Lozada, F.T., \& Craig, A. (2015). Parents' Emotion-Related Beliefs, Behaviours, and Skills Predict Children's Recognition of Emotion. Infant and Child Development, 24(1), 1-22.

Cherepinsky, V. (2011). Self-Reflective Grading: Getting Students to Learn from Their Mistakes. PRIMUS, 21(3), 294-301.

Diamond, A. (2010). The Evidence Base for Improving School Outcomes by Addressing the Whole Child and by Addressing Skills and Attitudes, Not Just Content. Early Education and Development, 21(5), 780-793.

Downing, J.J., \& Dyment, J.E. (2013). Teacher Educators' Readiness, Preparation, and Perceptions of Preparing Preservice Teachers in a Fully Online Environment: An Exploratory Study. Teacher Educator, 48(2), 96-109.

Duncan, D., \& Arthurs, L. (2012). Improving Student Attitudes about Learning Science and Student Scientific Reasoning Skills. Astronomy Education Review, 11(1), 101-130.

Ells, C. (2011). Communicating Qualitative Research Study Designs to Research Ethics Review Boards. Qualitative Report, 16(3), 881-891.

Ennen, N., Stark, E., \& Lassiter, A. (2015). The Importance of Trust for Satisfaction, Motivation, and Academic Performance in Student Learning Groups. Social Psychology of Education: An International Journal, 18(3), 615-633.

Fernández-Pérez, M. (1989). Así enseña nuestra universidad. Salamanca: Hispagraphis.

Ferrandiz-Vindel, I.M., \& Jimenez, B. (2011). The Sociogram: The Analysis of Interpersonal Relationships in Higher Education. Journal of International Education Research, 7(5), 9-14.

Frederiksen, L.F., \& Beck, S. (2013). Didactical Positions and Teacher Collaboration: Teamwork between Possibilities and Frustrations. Alberta Journal of Educational Research, 59(3), 442461.

Hagenauer, G., \& Volet, S. (2014). "I Don't Think I Could, You Know, Just Teach without Any Emotion": Exploring the Nature and Origin of University Teachers' Emotions. Research Papers in Education, 29(2), 240-262.

Harrinson, H. (2014). The Future of Science Teacher Education. Education in Science, 25(8), 6-7.

Hendry, G., \& Tomitsch, M. (2014). Implementing an Exemplar-Based Approach in an Interaction Design Subject: Enhancing Students' Awareness of the Need to Be Creative. International Journal of Technology and Design Education, 24(3), 337-348.

Hortigüela, D., Pérez-Pueyo, Á., \& López-Pastor, V. (2015). Student involvement and management of students' workload in formative assessment in higher education. Relieve: revista electrónica de investigación y evaluación educativa, 21(1), 1-5. doi:10.7203/relieve.21.1.5171

Jang, S.H. (2011). Assessing College Students' Perceptions of a Case Teacher's Pedagogical Content Knowledge Using a Newly Developed Instrument. Higher Education: The International Journal of Higher Education and Educational Planning, 61(6), 663-678.

Jensen, J. (2011). Higher Education Faculty versus High School Teacher: Does Pedagogical Preparation Make a Difference? Bioscene: Journal of College Biology Teaching, 37(2), 30-36.

Köpsén, S. (2014). How Vocational Teachers Describe Their Vocational Teacher Identity. Journal of Vocational Education and Training, 66(2), 194-211.

Lebec, M.T., \& Kesteloot, L. (2015). Overcoming Content-Associated Challenges Using AttentionFocused Methods. Journal on Excellence in College Teaching, 26(3), 25-56.

Lindell, M. (2006). From Formulation to Realisation: The Process of Swedish Reform in Advanced 
Vocational Education. Education \& Training, 48(4), 222-240.

Marcelo, C., \& Vaillant, D. (2009). Desarrollo profesional docente: ¿Cómo se aprende a enseñar? Madrid: Narcea.

Michie, M. (2013). Methodological Pluralism and Narrative Inquiry. Cultural Studies of Science Education, 8(3), 517-520.

Ní Chróinín, D., \& O'Sullivan, M. (2014). From Initial Teacher Education through Induction and Beyond: A Longitudinal Study of Primary Teacher Beliefs. Irish Educational Studies, 33(4), 451-466.

Palacios Picos, A., \& López-Pastor, V. M. (2013). Haz lo que yo digo pero no lo que yo hago: sistemas de evaluación del alumnado en la formación inicial del profesorado. Revista de Educación, 36(1), 279-305.

Pang, K. (2010). Creating Stimulating Learning and Thinking Using New Models of Activity-Based Learning and Metacognitive-Based Activities. Journal of College Teaching \& Learning, 7(4), 29-38.

Poole, G. (2013). The Coming and Going: The Work of Educational Developers When Admission Criteria and Desired Outcomes Change Simultaneously. International Journal for Academic Development, 18(4), 344-355.

Powers, L., Alhussain, R., Averbeck, C., \& Warner, A. (2012). Perspectives on Distance Education and Social Media. Quarterly Review of Distance Education, 13(4), 241-245.

Robinson, S., \& Mendelson, A.L. (2012). A Qualitative Experiment: Research on Mediated Meaning Construction Using a Hybrid Approach. Journal of Mixed Methods Research, 6(4), 332-347.

Saldaña, J. (2009). The Coding Manual for Qualitative Researchers. Thousand Oaks, CA: Sage.

Strauss, A., \& Corbin J. (2002). Bases de la investigación cualitativa: técnicas y procedimientos para desarrollar la teoría fundamentada. Colombia: Editorial Universidad de Antioquia.

Svensson, L., \& Doumas, K (2013). Contextual and Analytic Qualities of Research Methods Exemplified in Research on Teaching. Qualitative Inquiry, 19(6), 441-450.

Talburt, S., (2004). Ethnographic Responsibility without the "Real". Journal of Higher Education, 75(1), 80-87.

Tejedor, F. J. (1998). Las estrategias utilizadas por los profesores universitarios para la evaluación del aprendizaje de los alumnos. Madrid: CIDE.

Tortorella, G.L., Viana, S., \& Fettermann, D. (2015). Learning Cycles and Focus Groups: A Complementary Approach to the A3 Thinking Methodology. Learning Organization, 22(4), 229-240.

Van der Klink, M., Boon, J., \& Schlusmans, K. (2007). Competences and Vocational Higher Education: Now and in Future. European Journal of Vocational Training, 40(1), 67-82.

Välimaa, J., \& Nokkala, T. (2014). The Dimensions of "Social Dynamics" in Comparative Studies on Higher Education. Higher Education: The International Journal of Higher Education and Educational Planning, 67(4), 423-437.

Waterman, M., Weber, J., Pracht, C., Conway, K., Kunz, D., Evans, B., ... Starrett, D. (2010). Preparing Scholars of Teaching and Learning Using a Model of Collaborative Peer Consulting and Action Research. International Journal of Teaching and Learning in Higher Education, 22(2), 140-155. 
